\title{
Applications of Shortest Path Algorithms to Matrix Scalings $\star$
}

Dedicated to Professor F.L. Bauer on the occasion of his 60 th birthday

\author{
M. v. Golitschek ${ }^{1}$ and H. Schneider ${ }^{2}$ \\ ${ }^{1}$ Institut für Angewandte Mathematik und Statistik, D-8700 Würzburg (Fed. Rep.) \\ 2 Department of Mathematics, University of Wisconsin, Madison, Wisconsin 53706, USA
}

\begin{abstract}
Summary. A symmetric scaling of a nonnegative, square matrix $A$ is a matrix $X A X^{-1}$, where $X$ is a nonsingular, nonnegative diagonal matrix. By associating a family of weighted directed graphs with the matrix $A$ we are able to adapt the shortest path algorithms to compute san optimal scaling of $A$, where we call a symmetric scaling $A^{\prime}$ of $A$ optimal if it minimizes the maximum of the ratio of non-zero elements.
\end{abstract}

Subject Classifications: AMS(MOS): 65F35; CR: 5.14.

\section{Introduction}

The scaling of matrices has been considered from several points of view. At one extreme some papers concentrate on theoretical characterizations, e.g. [12] or $[18]$, and at the other papers report on the efficiency of algorithms, e.g. Tomlin [22]. This paper falls between these extremes for we are concerned with the development of algorithms which may however be stated as mathematical theorems.

We now briefly describe our paper. After some preliminaries in Sect. 2 we turn in Sect. 3 to the construction of an algorithm for a problem solved theoretically in [12]: Given nonnegative square matrices $A, B, C$ when does there exist a symmetric scaling $A^{\prime}=X A X^{-1}$ of $A$ (where $X$ is diagonal) for which $C \leqq A^{\prime} \leqq B$ ? Our algorithm is related to Karp's algorithm for finding the minimum cycle mean in a weighted directed graph, see [14], and its first version was developed by one of us in $[10,11]$ and is there applied in the consideration of asymmetric scalings: $A^{\prime}=X A Y$. In [11] the present form of the algorithm is given and is there used for the approximation of bivariate

* The first author was partially supported by the Deutsche Forschungsgemeinschaft under grant GO 270/3, the second author by the U.S. National Science Foundation under grand MCS-8026132 
functions by univariate ones. Here we apply the algorithm to give an algorithmic answer to the problem described above.

Our main results may be found in Sects. 4 and 5. We again turn to a problem previously solved theoretically, see [18, Theorem 6]: Given a nonnegative matrix $A$ characterize the symmetric scalings $A^{\prime}$ which minimize the maximum of the ratio of non-zero elements. By associating a family of weighted directed graphs with the matrix $A$ we are able to adapt our algorithm to compute an optimal scaling $A^{\prime}$. In Sect. 6 we then give a new proof of the theorem in [18] based on this algorithm.

By means of the bipartite expansion of a directed graph it is possible to obtain from any result on symmetric scalings a corresponding result on asymmetric scalings, see [19, Sect. 3]. The asymmetric formulation of some of our results is given in Sect. 7 .

\section{Notations}

In this paper, $n$ will be a fixed positive integer,

$$
V=\{1 ; 2 ; \ldots ; n\} \text { and } E \subseteq V \times V, \quad E \neq \emptyset .
$$

By $G=(V, E)$ we denote the directed graph with vertices $V$ and $\operatorname{arcs} e=(i, j) \in E$.

A sequence $\beta=\left(e_{1}, \ldots, e_{k}\right), k \geqq 1$, of $\operatorname{arcs} e_{s}=\left(i_{s}, j_{s}\right) \in E, s=1, \ldots, k$, is a path (of length $|\beta|=k$ from $i_{1}$ to $j_{k}$ ) if $j_{s}=i_{s+1}, s=1, \ldots, k-1$.

A path $\beta$ is closed if $i_{1}=j_{k}$, and a closed path $\beta$ is a directed cycle if $i_{1}, \ldots, i_{k}$ are pairwise distinct. We put

$$
\begin{aligned}
W_{j}^{k}: & =\{\beta: \beta \text { is a path of length } k \text { ending at } j\} \\
W^{k}: & =\{\beta: \beta \text { is a path of length } k\}, \\
W_{j}: & =\{\beta: \beta \text { is a path ending at } j\}, \\
Y: & =\{\beta: \beta \text { is a closed path }\} \\
Z: & =\{\beta: \beta \text { is a directed cycle }\}
\end{aligned}
$$

Finally, we say that $(V, E, d)$ is a weighted directed graph with the weight function $d: E \rightarrow \mathbb{R}$.

The weight $d(\beta)$ of a path $\beta=\left(e_{1}, \ldots, e_{k}\right)$ is defined by

and

$$
d(\beta):=\sum_{s=1}^{k} d\left(e_{s}\right)
$$

$$
\lambda^{*}:=\min _{\beta \in X} \frac{d(\beta)}{|\beta|}=\min _{\beta \in Z} \frac{d(\beta)}{|\beta|}
$$

is called the minimum cycle mean in the weighted directed graph $(V, E, d)$.

Notice that loops $\beta=(i, i) \in E$ are elements of $Z$. 


\section{Fundamental Results}

We now turn to the problem discussed in the introduction.

Problem 1. Let $a_{i j} \in \mathbb{R}, b_{i j} \in \mathbb{R} \cup\{\infty\}, c_{i j} \in \mathbb{R} \cup\{-\infty\}, i, j=1,2, \ldots, n$. Do there exist $x_{i} \in \mathbb{R}, i=1, \ldots, n$, for which

$$
c_{i j} \leqq x_{i}+a_{i j}-x_{j} \leqq b_{i j}, \quad i, j=1, \ldots, n ?
$$

A special case of Problem 1 is the following.

Problem 2. Let $E \subseteq V \times V, d_{i j} \in \mathbb{R}$ for $(i, j) \in E$. Do there exist $x_{i} \in \mathbb{R}, i=1, \ldots, n$, for which

$$
0 \leqq x_{i}+d_{i j}-x_{j}, \quad(i, j) \in E ?
$$

Our next lemma shows that Problem 1 is equivalent to Problem 2, and can be solved with the same $x_{i}, i=1, \ldots, n$, provided that $E$ and the $d_{i j}$ are properly chosen.

Lemma 1. Let $a_{i j}, b_{i j}, c_{i j}$ be as in Problem 1. Let

$$
\begin{aligned}
d_{i j} & :=\min \left\{a_{i j}-c_{i j} ; b_{j i}-a_{j i}\right\}, \quad i, j=1, \ldots, n, \\
E & :=\left\{(i, j): d_{i j} \neq \infty\right\} .
\end{aligned}
$$

Then $x=\left(x_{1}, \ldots, x_{n}\right) \in \mathbb{R}^{n}$ solves (3.1) if and only if $x$ solves (3.2).

Proof. The inequalities (3.1) are equivalent to the $2 n^{2}$ inequalities

$$
\begin{array}{ll}
0 \leqq x_{i}+a_{i j}-c_{i j}-x_{j}, & i, j=1, \ldots, n \\
0 \leqq x_{i}+b_{j i}-a_{j i}-x_{j}, & i, j=1, \ldots, n
\end{array}
$$

where the last relations are obtained by interchanging $i$ and $j$. Thus we obtain the equivalent form

$$
0 \leqq x_{i}+d_{i j}-x_{j}, \quad i, j=1, \ldots, n \text {. }
$$

Since the inequalities above are trivially satisfied if $d_{i j}=\infty$, they are equivalent to $(3.2)$.

Our algorithm for solving Problem 2 (and thus Problem 1) is based on the following theorem which states that Problem 2 is equivalent to the negative cycle problem in weighted directed graphs.

Theorem 1. Let $E \subseteq V \times V, d(e):=d_{i j}$ for $e=(i, j) \in E$.

(a) If there is a directed cycle $\beta$ of negative weight $d(\beta)$ in the weighted directed graph $(V, E, d)$, then Problem 2 has no solution.

(b) Otherwise the vector $x=\left(x_{1}, \ldots, x_{n}\right) \in \mathbb{R}^{n}$ defined by

$$
x_{j}:=\min \left\{0 ; d(\beta): \beta \in \bigcup_{k=1}^{n} W_{j}^{k}\right\}, \quad j=1, \ldots, n
$$

is a solution of (3.2). 
Proof. (a) Let $x_{i} \in \mathbb{R}, i=1, \ldots, n$, and put $d_{i j}^{\prime}:=x_{i}+d_{i j}-x_{j},(i, j) \in E$. Then for any directed cycle $\beta$ in $(V, E), d(\beta)=d^{\prime}(\beta)$. Hence Problem 2 has no solution if $d(\beta)<0$ for some directed cycle $\beta$ in $(V, E)$.

(b) Let $x_{j}$ be defined by $(3.5), j=1, \ldots, n$. Since there is no directed cycle of negative weight in $(V, E, d)$, we have

$$
x_{j}=\min \left\{0 ; d(\beta): \beta \in W_{j}\right\}, \quad j=1, \ldots, n .
$$

Suppose that $x_{i}=d\left(\beta_{i}\right)$ where $\beta_{i} \in W_{i}$. If $(i, j) \in E$ and $\gamma$ is the path $\beta_{i}$ followed by the $\operatorname{arc}(i, j)$, then $d(\gamma)=x_{i}+d_{i j} \geqq x_{j}$, since $\gamma \in W_{j}$. Hence $x_{i}+d_{i j}-x_{j} \geqq 0$ if $(i, j) \in E$.

Corollary 1. The minimum cycle mean $\lambda^{*}$ of the weighted directed graph $(V, E, d)$ is the largest of the numbers $\lambda$ for which the inequalities

have a solution $x \in \mathbb{R}^{n}$.

$$
x_{i}+d_{i j}-x_{j} \geqq \lambda, \quad(i, j) \in E
$$

Proof. Applying Theorem 1 for the weights $d_{i j}^{\prime}:=d_{i j}-\lambda,(i, j) \in E$, we realize that (3.6) has a solution $x$ iff

$$
d^{\prime}(\beta)=d(\beta)-\lambda|\beta| \geqq 0 \quad \text { for all } \beta \in Z,
$$

and hence iff $\lambda \leqq \min _{\beta \in Z} \frac{d(\beta)}{|\beta|}=\lambda^{*}$.

Remark 1 . The above results are partly well-known and have already appeared in the literature in various guises and forms, see e.g. [11, Theorem 1.1] and the detailed theoretical investigations of Problem 1 in [12]. The equivalence of Problem 1 and Problem 2, but in particular our Theorem 1 play an essential role in algorithms which are concerned with the planning and scheduling in MPM networks (MPM = Metra-Potential-Method), see e.g. [23, Part III, $\S 1.31$ and $\S 1.32]$.

Lemma 1 and Theorem 1 give the following information: Any algorithm which determines if a weighted directed graph $(V, E, d)$ contains a directed cycle of negative weight can be applied to Problem 1 and Problem 2. Efficient algorithms of this kind have been constructed by Ford and Bellman, by Yen, by Dantzig, Blattner, Rao, and by others, see e.g. [15].

Our Algorithm 1 below is a modification of the famous Ford-Bellman algorithm and a first version of Karp's algorithm for finding the minimum cycle mean in a weighted directed graph. Algorithm 1 has been suggested in the present form in [11].

\section{Algorithm 1 (The Minimum Cycle Mean Problem)}

Step 0. We suppose that the forward and backward stars of each vertex $j \in V$ are non-empty. Otherwise, we may erase such vertices.

Set $x_{j}(0):=0$ for $j \in V, J(0):=\{1 ; \ldots, n\}, k:=1$. 
Step 1. For $j \in V$, compute

$$
\begin{aligned}
x_{j}(k) & :=\min \left\{x_{j}(k-1) ; x_{i}(k-1)+d_{i j}: i \in J(k-1),(i, j) \in E\right\} ; \\
J(k) & :=\left\{j \in V: x_{j}(k)<x_{j}(k-1)\right\} .
\end{aligned}
$$

For any $j \in J(k)$, choose one of the indices $i \in J(k-1)$, for which the minimum in (3.7) is attained, and call it $P_{j k}$.

If $J(k)=\emptyset$, put

$$
x_{j}:=x_{j}(k), \quad j=1, \ldots, n \text {. Stop. }
$$

If $k=n$ and $J(n) \neq \emptyset$, Stop (Problem 2 has no solution).

If $k<n$ and $J(k) \neq \emptyset$, put $k:=k+1$ and return to Step 1 .

Step 2. Suppose that all sets $J(k), k=1, \ldots, n$, are non-empty.

(a) Compute the minimum cycle mean $\lambda^{*}$ of $(V, E, d)$ by

$$
\lambda^{*}=\min _{j \in J(n)} \max _{0 \leqq k \leqq n-1} \frac{x_{j}(n)-x_{j}(k)}{n-k} .
$$

(b) Choose any $j \in J(n)$ for which the minimum in (3.9) is attained. Construct the sequence of vertices by the recurrence

$$
j_{n}:=j, \quad j_{k-1}:=P_{j_{k}, k} \quad \text { for } k=n, n-1, \ldots
$$

until the first time equality $j_{r}=j_{s}$ occurs for some $r$ and $s, 0 \leqq r<s \leqq n$. Then

$$
\beta^{*}:=\left\{\left(j_{r}, j_{r+1}\right), \ldots,\left(j_{s-1}, j_{s}\right)\right\}
$$

is a directed cycle in $(V, E)$ with $d\left(\beta^{*}\right) /\left|\beta^{*}\right|=\lambda^{*}$.

Remark 2. The properties of Algorithm 1 have been studied in [11] under the assumption that the directed graph $(V, E)$ has no loops. In our paper, loops are admitted and belong to the set $Z$ of directed cycles in $(V, E)$. But it turns out easily that these loops don't cause any additional difficulties in the proofs of [11].

In more detail, the following properties of Algorithm 1 have been proved in [11; Theorem 2.1, pp. $68 \mathrm{ff}$.$] .$

Theorem 2. (a) The minimum cycle mean $\lambda^{*}$ of $(V, E, d)$ is non-negative if and only if there exists an index $k, 1 \leqq k \leqq n$, for which the set $J(k)$ is empty.

(b) If $J(k)$ is empty for some $k$, then the numbers $x_{j}, j=1, \ldots, n$, defined by (3.8) and by (3.5) are the same, and therefore solve Problem 2.

(c) If all sets $J(k), k=1, \ldots, n$, are non-empty, then $\lambda^{*}$ can be computed by (3.9) and the directed cycle $\beta^{*}$ of form (3.11) constructed in Step 2 of Algorithm 1 has the property $d\left(\beta^{*}\right) /\left|\beta^{*}\right|=\lambda^{*}$.

The computation of the minimum cycle mean $\lambda^{*}$ and of an optimal directed cycle $\beta^{*}$ in weighted directed graphs will play an important role in the Sects. 4-7. For the solution of Problem 2 we need less than Algorithm 1 achieves. If we want to solve Problem 2 (see also [11; Sect. 4]) then we can simplify Algorithm 1 to the following. 
Algorithm 2 (Problem 1 and Problem 2)

0. Perform Step 0 of Algorithm 1.

1. Perform Step 1 of Algorithm 1 without noting the indices $P_{j k}$. If all sets $J(k), k=1, \ldots, n$, are non-empty then Problem 2 has no solution, else the numbers $x_{j}$ defined by (3.8) solve Problem 2.

\section{Symmetric Scaling of Matrices}

Let $A=\left(A_{i j}\right) \neq 0$ be a non-negative $n \times n$-matrix. The matrices of the form $X A X^{-1}$ with $X \in D_{n}^{+}$are called symmetric scalings of $A$ where $D_{n}^{+}$is the set of non-negative, non-singular, diagonal $n \times n$-matrices. The measure of a symmetric scaling $X A X^{-1}$ is given by

$$
\alpha(X)=\frac{\max _{(i, j) \in E_{0}}\left(X A X^{-1}\right)_{i j}}{\min _{(k, l) \in E_{0}}\left(X A X^{-1}\right)_{k l}}
$$

where $E_{0}:=\left\{(i, j): A_{i j}>0\right\}$.

It is the main purpose of our paper to present an efficient algorithm for the following Symmetric Scaling Problem.

Problem 3. Find the infimum

$$
\alpha:=\inf _{X \in D_{n}^{+}} \alpha(X)
$$

and a diagonal matrix $X^{*} \in D_{n}^{+}$with $\alpha\left(X^{*}\right)=\alpha$.

We put

$$
a_{i j}:=\log A_{i j}, \quad(i, j) \in E_{0}
$$

and define the set $\Omega \in \mathbb{R}^{2}$ by

$$
\Omega:=\left\{\begin{array}{l}
(m, M): \text { there exist } x_{i} \in \mathbb{R}, i=1, \ldots, n, \text { for which } \\
m \leqq x_{i}+a_{i j}-x_{j} \leqq M,(i, j) \in E_{0} .
\end{array}\right.
$$

Let $X=\operatorname{diag}\left(X_{1}, \ldots, X_{n}\right) \in D_{n}^{+}$and $x_{i}:=\log X_{i}, i=1, \ldots, n$. Taking the logarithm of (4.1) leads to

$$
\log \alpha(X)=\max _{(i, j) \in E_{0}}\left(x_{i}+a_{i j}-x_{j}\right)-\min _{(i, j) \in E_{0}}\left(x_{i}+a_{i j}-x_{j}\right)
$$

and thus to the following additive form of the Problem 3.

Problem 3'. Find the infimum

$$
\log \alpha=\inf \{M-m:(m, M) \in \Omega\}
$$

and numbers $m^{*}, M^{*}, x_{1}^{*}, \ldots, x_{n}^{*}$ such that $M^{*}-m^{*}=\log \alpha$ and

$$
m^{*} \leqq x_{i}^{*}+a_{i j}-x_{j}^{*} \leqq M^{*}, \quad(i, j) \in E_{0} .
$$

The following lemma is obviously true. 
Lemma 2. The real numbers $x_{1}^{*}, \ldots, x_{n}^{*}$ solve (4.4) with $M^{*}-m^{*}=\log \alpha$ if and only if $\alpha\left(X^{*}\right)=\alpha$ for

$$
X^{*}=\operatorname{diag}\left(e^{x^{*}}, \ldots, e^{x_{n}^{*}}\right) \text {. }
$$

After associating with the matrix $A \neq 0$ a family of weighted directed graphs $G^{s}, s \in \mathbb{R}$, it will turn out that the Problem 3 and the Problem $3^{\prime}$ are also equivalent to the Problem $3^{\prime \prime}$ below which will be studied and solved by graph theoretical methods. But first, we need some notations.

We put

$$
V:=\{1, \ldots, n\} ; \quad E:=E_{0} \cup\left\{(i, j):(j, i) \in E_{0}\right\}
$$

and denote by $Z$ the set of all directed cycles in the directed graph $G=(V, E)$. For any real number $s$, we define the weight function $d^{s}: E \rightarrow \mathbb{R}$ by

$$
d_{i j}^{s}:=\min *\left\{a_{i j}-s ; s-a_{j i}\right\}, \quad(i, j) \in E
$$

where in (4.5) the notation min* indicates that we set in (4.5) $a_{i j}-s=+\infty$ if $(i, j) \notin E_{0}$ and $s-a_{j i}=+\infty$ if $(j, i) \notin E_{0}$. We put

$$
\begin{aligned}
& E_{1}^{s}:=\left\{(i, j) \in E: a_{i j}-s=d_{i j}^{s}\right\} \\
& E_{2}^{s}:=\left\{(i, j) \in E: s-a_{j i}=d_{i j}^{s}\right\} \\
& E_{3}^{s}:=E_{1}^{s} \cap E_{2}^{s} .
\end{aligned}
$$

For any directed cycle $\beta \in Z$ we denote its number of arcs in $E_{r}^{s}, r=1,2,3$, by $N_{r}^{s}(\beta):=\left|\beta \cap E_{r}^{s}\right|$ and put

$$
\begin{aligned}
& n_{1 s}(\beta):=N_{1}^{s}(\beta)+N_{3}^{s}(\beta)-N_{2}^{s}(\beta), \\
& n_{2 s}(\beta):=N_{1}^{s}(\beta)-N_{3}^{s}(\beta)-N_{2}^{s}(\beta) .
\end{aligned}
$$

Finally, with each $s \in \mathbb{R}$ we associate the weighted directed graph $G^{s}=\left(V, E, d^{s}\right)$ and denote the minimum cycle mean in $G^{s}$ by $\varphi(s)$, i.e.

$$
\varphi(s):=\min _{\beta \in Z} d^{s}(\beta) /|\beta|,
$$

and by $\beta_{s} \in Z$ one of the directed cycles in $(V, E)$ for which the minimum in (4.6) is attained, i.e.

$$
d^{s}\left(\beta_{s}\right) /\left|\beta_{s}\right|=\varphi(s)
$$

Since $d_{i j}^{s} \leqq-d_{j i}^{s},(i, j) \in E$, the minimum cycle mean $\varphi(s)$ of $G^{s}$ is non-positive for each $s$.

Problem $3^{\prime \prime}$. Find the supremum

$$
\sup _{s \in R} \varphi(s)
$$

and a number $s^{*}$ for which the supremum is attained.

The next theorem will show that the Problems 3, 3', 3' are equivalent, that the supremum in (4.8) and thus the infima in the Problems 3 and $3^{\prime}$ are attained, and how the optimal numbers $x_{1}^{*}, \ldots, x_{n}^{*}$ of the Problem $3^{\prime}$ are connected with the minimum cycle mean problem. 
Theorem 3. 1. There exists an $s^{*} \in \mathbb{R}$ for which $\varphi\left(s^{*}\right)=\sup \varphi(s)$.

2. The supremum (4.8) is attained at a number $s^{*}$ if and only if the infimum (4.3) of the Problem 3' is attained by

$$
m^{*}:=s^{*}+\varphi\left(s^{*}\right), \quad M^{*}:=s^{*}-\varphi\left(s^{*}\right) .
$$

Additionally, any solution $x_{1}^{*}, \ldots, x_{n}^{*}$ of

$$
x_{i}^{*}+d_{i j}^{s^{*}}-x_{j}^{*} \geqq \varphi\left(s^{*}\right), \quad(i, j) \in E
$$

is also a solution of (4.4), and conversely. And

$$
\log \alpha=M^{*}-m^{*}=-2 \varphi\left(s^{*}\right)
$$

Proof. 1. The set $E_{3}^{s}=\left\{(i, j) \in E: a_{i j}-s=s-a_{j i}\right\}$ is empty except for finitely many numbers $s$, say except for $s_{1}<s_{2}<\ldots<s_{q}$. For any $\beta \in Z, d^{s}(\beta)$ is a continuous function in $s$ and linear in the intervals $\left(-\infty, s_{1}\right),\left(s_{1}, s_{2}\right), \ldots,\left(s_{q}, \infty\right)$. Since the number of directed cycles $\beta \in Z$ is finite, $\varphi$ is a piecewise linear continuous function consisting of only finitely many linear pieces. But as $\varphi(s) \leqq 0$ for all $s$, $\varphi$ attains its maximal value.

2. We need the following lemma.

Lemma 3. For any $s,|\varphi(s)|=-\varphi(s)$ is the smallest of all non-negative numbers $\rho$ for which

$$
s-\rho \leqq x_{i}+a_{i j}-x_{j} \leqq s+\rho, \quad(i, j) \in E_{0}
$$

has a solution $\left(x_{1}, \ldots, x_{n}\right)$.

Proof of Lemma 3. It follows from Corollary 1 that $\varphi(s)$ is the largest of all numbers $\lambda$ for which

$$
x_{i}+d_{i j}^{s}-x_{j} \geqq \lambda, \quad(i, j) \in E
$$

has a solution $\left(x_{1}, \ldots, x_{n}\right)$. But by Lemma $1,(4.12)$ and (4.13) are equivalent if $\rho$ $=-\lambda$.

Since $\left|\varphi\left(s^{*}\right)\right|=\min |\varphi(s)|$, the second part of Theorem 3 follows directly from Lemma 3 and the equivalence of (4.12) and (4.13) if $\rho=-\lambda$.

Since the Problems 3, 3', $3^{\prime \prime}$ are equivalent we shall solve the Symmetric Scaling Problem 3 by characterizing and computing the maximal points $s^{*}$ of the function $\varphi$. For this purpose, we first have to study the essential properties of $\varphi$ in our next theorem.

Theorem 4. 1. $\varphi$ is continuous, non-positive, and piecewise linear consisting of finitely many linear pieces.

2. $\varphi \in \operatorname{Lip}_{1}$ 1, i.e. $|\varphi(s)-\varphi(t)| \leqq|s-t|$ for all $s, t$.

3. $\varphi$ is concave.

4. $\varphi$ satisfies the inequality

$$
\left|\beta_{s}\right|(\varphi(t)-\varphi(s)) \leqq \min _{r=1,2}(s-t) n_{r s}\left(\beta_{s}\right)
$$

for any numbers $s$ and $t$ and any $\beta_{s} \in Z$ satisfying (4.7). 
Proof. 1) has already been proved in the proof of Theorem 3.

2) Since $-\left|\beta_{s}\right| \leqq n_{2 s}\left(\beta_{s}\right) \leqq n_{1 s}\left(\beta_{s}\right) \leqq\left|\beta_{s}\right|$, it follows from (4.14) that $\varphi(t)$ $-\varphi(s) \leqq|t-s|$ for all $s$ and $t$. Hence, $\varphi \in \operatorname{Lip}_{1} 1$.

3) Suppose that $s_{0}<s_{1}$ and $s:=(1-u) s_{0}+u s_{1}$ for a fixed number $u$, $0<u<1$. It follows from (4.14) with $t=s_{0}$ that

$$
\varphi\left(s_{0}\right) \leqq \varphi(s)-\left(s_{0}-s\right) n_{2 s}\left(\beta_{s}\right) /\left|\beta_{s}\right|
$$

and from (4.14) with $t=s_{1}$ that

Hence we have

$$
\varphi\left(s_{1}\right) \leqq \varphi(s)-\left(s_{1}-s\right) n_{1 s}\left(\beta_{s}\right) /\left|\beta_{s}\right| .
$$

$$
\varphi(s)-(1-u) \varphi\left(s_{0}\right)-u \varphi\left(s_{1}\right) \geqq u\left|\beta_{s}\right|^{-1}\left(s_{1}-s\right)\left[n_{1 s}\left(\beta_{s}\right)-n_{2 s}\left(\beta_{s}\right)\right] \geqq 0
$$

which shows that $\varphi$ is concave.

4) Let $s$ and $t$ be fixed. Then, for any $\beta \in Z$,

$$
|\beta| \varphi(t) \leqq d^{t}(\beta) \leqq d^{s}(\beta)+(s-t)\left(N_{1}^{s}(\beta) \pm N_{3}^{s}(\beta)-N_{2}^{s}(\beta)\right)
$$

which already proves (4.14) if we take $\beta=\beta_{s}$.

The next theorem gives an important characterization of the maximal points $s^{*}$ of the Problem $3^{\prime \prime}$ and thus (by Theorem 3 and the relation (4.3)) of the minimal solutions $\left(m^{*}, M^{*}\right)$ of the Problem $3^{\prime}$ and the minimum $\alpha$ of the Scaling Problem 3.

Theorem 5. The following three statements are equivalent.

(1) $\varphi$ attains its maximal value at $s^{*}$.

(2) There exist two (not necessarily distinct) directed cycles $\beta$ and $\beta^{\prime}$ in $Z$ for which the relations

and

$$
\varphi\left(s^{*}\right)=d^{s^{*}}(\beta) /|\beta|=d^{s^{*}}\left(\beta^{\prime}\right) /\left|\beta^{\prime}\right|
$$

are satisfied.

$$
n_{2 s^{*}}\left(\beta^{\prime}\right) \leqq 0 \leqq n_{1 s^{*}}(\beta)
$$

(3) For any number $s, s \neq s^{*}$, and any directed cycle $\beta_{s} \in Z$ satisfying (4.7), the integers $n_{1 s}\left(\beta_{s}\right)$ and $n_{2 s}\left(\beta_{s}\right)$ have the properties

$$
\begin{array}{ll}
n_{1 s}\left(\beta_{s}\right) \geqq n_{2 s}\left(\beta_{s}\right) \geqq 0 & \text { if } s>s^{*} \\
n_{2 s}\left(\beta_{s}\right) \leqq n_{1 s}\left(\beta_{s}\right) \leqq 0 & \text { if } s<s^{*} .
\end{array}
$$

Proof. (1) $\Rightarrow(3)$ : It follows from (4.14) that

which leads to (4.17).

$$
0 \leqq\left|\beta_{s}\right|\left(\varphi\left(s^{*}\right)-\varphi(s)\right) \leqq \min _{r=1,2}\left(s-s^{*}\right) n_{r s}\left(\beta_{s}\right)
$$

(3) $\Rightarrow(2)$ : Suppose that the Statement (3) is valid. Since the number $|Z|$ of directed cycles is finite, we find sequences $s_{k} \rightarrow s^{*}, s_{k}>s^{*}$, and $s_{k}^{\prime} \rightarrow s^{*}, s_{k}^{\prime}<s^{*}$, and two (not necessarily distinct) directed cycles $\beta$ and $\beta^{\prime}$ such that

$$
\varphi\left(s_{k}\right)=d^{s_{k}}(\beta) /|\beta|, \quad \varphi\left(s_{k}^{\prime}\right)=d^{s_{k}^{\prime}}\left(\beta^{\prime}\right) /\left|\beta^{\prime}\right|
$$


holds for all $k$. Because of (4.17) and (4.18),

$$
n_{1 s_{k}}(\beta) \geqq n_{2 s_{k}}(\beta) \geqq 0, \quad n_{2 s_{k}}\left(\beta^{\prime}\right) \leqq n_{1 s_{k}^{\prime}}\left(\beta^{\prime}\right) \leqq 0 .
$$

for all $k$. Hence, for $k \rightarrow \infty$, the continuity of $\varphi$ and $d_{i j}^{s}$ guarantees that (4.15) and (4.16) are valid.

$(2) \Rightarrow(1)$ : It follows from $(4.14)-(4.16)$ that

$$
\begin{array}{ll}
|\beta|\left(\varphi(t)-\varphi\left(s^{*}\right)\right) \leqq\left(s^{*}-t\right) n_{1 s^{*}}(\beta) \leqq 0 & \text { if } t>s^{*}, \\
\left|\beta^{\prime}\right|\left(\varphi(t)-\varphi\left(s^{*}\right)\right) \leqq\left(s^{*}-t\right) n_{2 s^{*}}\left(\beta^{\prime}\right) \leqq 0 & \text { if } t<s^{*}
\end{array}
$$

Hence, $\varphi(t) \leqq \varphi\left(s^{*}\right)$ for each $t$.

\section{An Algorithm for the Problems 3, 3', 3'}

We have seen in Lemma 2 and Theorem 3 that any solution $s^{*}, x_{1}^{*}, \ldots, x_{n}^{*}$ of Problem $3^{\prime \prime}$ and (4.10) leads by (4.9) to a solution $M^{*}-m^{*}=-2 \varphi\left(s^{*}\right)$ of Problem 3' (where (4.4) holds) and to a minimal symmetric scaling

with

$$
X^{*} A X^{*-1}, \quad X^{*}:=\operatorname{diag}\left(e^{x_{1}^{*}}, \ldots, e^{x_{n}^{*}}\right)
$$

$$
\alpha=\alpha\left(X^{*}\right)=e^{-2 \varphi\left(s^{*}\right)} .
$$

Hence it suffices to find a maximal point $s^{*}$ of $\varphi$ and numbers $x_{1}^{*}, \ldots, x_{n}^{*}$ satisfying (4.10). For this purpose, we suggest the algorithm below which is based on Theorem 5, Inequality (4.14) and on Algorithm 1 which will compute the minimum cycle mean $\varphi(s)$ of the weighted directed graph $G^{s}=\left(V, E, d^{s}\right)$ for finitely many real numbers $s$.

\section{Algorithm 3}

Step 0. Put $L:=-\infty, U:=+\infty$. ( $L$ and $U$ will serve as lower and upper bounds for a maximal point $s^{*}$ of $\varphi$.) Choose a real number $s$.

Step 1. Apply the Algorithm 1 to the weighted directed graph $G^{s}=\left(V, E, d^{s}\right)$ and compute $\varphi(s)$, one directed cycle $\beta_{s}$ satisfying (4.7), and the integers $n_{r s}$ $:=n_{r s}\left(\beta_{s}\right), r=1,2$.

a) If $n_{2 s} \leqq 0 \leqq n_{1 s}$, put $s^{*}:=s$ and goto Step 2 .

b) If $n_{1 s}<0$, put $L:=s$. If $n_{2 s}>0$, put $U:=s$.

c) Choose the new number $s$ as follows.

if $-\infty<L \leqq U<+\infty$, and

$$
s:=s_{L U}:=L+\frac{\left|\beta_{L}\right|(U-L) n_{2 U}+\left|\beta_{L}\right|\left|\beta_{U}\right|(\varphi(U)-\varphi(L))}{\left|\beta_{L}\right| n_{2 U}-\left|\beta_{U}\right| n_{1 L}}
$$

$$
s:=s_{L U}:= \begin{cases}L+\left|\beta_{L}\right| \varphi(L) / n_{1 L} & \text { if } U=+\infty \\ U+\left|\beta_{U}\right| \varphi(U) / n_{2 U} & \text { if } L=-\infty\end{cases}
$$


d) If $s_{L U}=L$ or $s_{L U}=U$, put $s^{*}:=s_{L U}$ and go to Step 2. Else return to Step 1.

Step 2. $s^{*}$ is a maximal point of $\varphi$.

Apply the Algorithm 1 to the weighted directed graph $\left(V, E, d^{*}\right)$ where the weight function $d^{*}$ is defined by

$$
\left.d_{i j}^{*}:=d_{i j}^{s^{*}}-\varphi\left(s^{*}\right)=\min * a_{i j}-s^{*} ; s^{*}-a_{j i}\right\}-\varphi\left(s^{*}\right),
$$

$(i, j) \in E$. Since the minimum cycle mean of $\left(V, E, d^{*}\right)$ is equal to zero, the event (3.8) must occur for some index $k, 1 \leqq k \leqq n$, and the numbers $x_{j}^{*}:=x_{j}(k), j$ $=1, \ldots, n$, which we obtain from (3.8), are the desired solution of the inequalities (4.10).

Remark 3. 1. If the events (a) or (d) of Step 1 occur, then the inequalities (4.14) imply that $s$ is a maximal point of $\varphi$.

2. The number $s_{L U}$ in Step 1 is the unique point where the straight lines

$$
\begin{aligned}
& y_{1}(t):=\left\{\begin{array}{ll}
\varphi(L)-(t-L) n_{1 L} /\left|\beta_{L}\right|, & \text { if } L>-\infty \\
0, & \text { if } L=-\infty
\end{array}\right\} t \in \mathbb{R} \\
& y_{2}(t):=\left\{\begin{array}{ll}
\varphi(U)-(t-U) n_{2 U} /\left|\beta_{U}\right|, & \text { if } U<+\infty \\
0, & \text { if } U=+\infty
\end{array}\right\} t \in \mathbb{R}
\end{aligned}
$$

intersect. Hence, $L \leqq s_{L U} \leqq U$.

3. The above Algorithm 3 finds a maximal point $s^{*}$ of $\varphi$ in finitely many iterations. That follows at once from the inequalities (4.14).

\section{An Explicit Characterization of the Minimum $\alpha$}

In [18; Theorem 6], Rothblum and Schneider haved proved a characterization of the minimal value $\alpha$ of the Problem 3. We shall now show how this result follows from our Theorem 5 .

Let the notation be the same as in Sect. 4. More exactly, let $A=\left(A_{i j}\right) \neq 0$ be a non-negative $n \times n$-matrix, $V:=\{1, \ldots, n\}, E_{0}:=\left\{(i, j): A_{i j}>0\right\}$, and

$$
a_{i j}:=\log A_{i j} \quad \text { for }(i, j) \in E_{0} .
$$

We now consider another directed graph, $G_{0}:=\left(V, E_{0}\right)$. In this section, $G_{0}$ and the (oriented) cycles $c$ in $G_{0}$ play the essential role.

Definition. $c=c^{+} \cup c^{-}=\left\{e_{1}, \ldots, e_{k}\right\} \subseteq E_{0}, k \geqq 1$, is called a cycle in $G_{0}$ if there exist $k$ distinct vertices in $V$, say $i_{1}, \ldots, i_{k}$, such that

$$
e_{r}=\left\{\begin{array}{ll}
\left(i_{r}, i_{r+1}\right), & \text { if } e_{r} \in c^{+} \\
\left(i_{r+1}, i_{r}\right), & \text { if } e_{r} \in c^{-}
\end{array}\right\} r=1, \ldots, k
$$

where we set $i_{k+1}:=i_{1}$. By $Z_{0}$ we denote the set of all cycles $c=c^{+} \cup c^{-}$in $G_{0}$. 
We associate with any arc $e=(i, j) \in E_{0}$ its weight $D(e):=a_{i j}$ and with any cycle $c=c^{+} \cup c^{-} \in Z_{0}$ its weight

$$
D(c):=\sum_{e \in c^{+}} D(e)-\sum_{e \in c^{-}} D(e)
$$

We put $N(c):=\left|c^{+}\right|-\left|c^{-}\right|$and

$$
\begin{aligned}
& C^{0}\left(G_{0}\right):=\left\{c \in Z_{0}: N(c)=0\right\}, \\
& C^{2}\left(G_{0}\right):=\left\{\left(c_{1}, c_{2}\right) \in Z_{0} \times Z_{0}: N\left(c_{2}\right)<0<N\left(c_{1}\right)\right\} .
\end{aligned}
$$

Finally, with any cycle $c \in Z$, we associate the directed cycle $\bar{c} \in Z$ in $G=(V, E)$ defined by

$$
\bar{c}:=\left\{(i, j) \in E:(i, j) \in c^{+}\right\} \cup\left\{(j, i) \in E:(i, j) \in c^{-}\right\} .
$$

Here, $E$ and $Z$ are defined as in Sect. 4.

We then have for each cycle $c=c^{+} \cup c^{-} \in Z_{0}$ and each $s$

$$
d^{s}(\vec{c})=\sum_{(i, j) \in c^{+}} \min *\left\{a_{i j}-s ; s-a_{j i}\right\}+\sum_{(i, j) \in c^{-}} \min ^{*}\left\{a_{j i}-s ; s-a_{i j}\right\}
$$

and hence

$$
|\bar{c}| \varphi(s)=|c| \varphi(s) \leqq d^{s}(\vec{c}) \leqq D(c)-s N(c)
$$

and

$$
\begin{aligned}
& \varphi(s) \leqq D(c) /|c| \quad \text { if } c \in C^{0}\left(G_{0}\right), \\
& \varphi(s) \leqq \frac{D\left(c_{2}\right) N\left(c_{1}\right)-D\left(c_{1}\right) N\left(c_{2}\right)}{\left|c_{2}\right| N\left(c_{1}\right)-\left|c_{1}\right| N\left(c_{2}\right)}=: I\left(c_{1}, c_{2}\right)
\end{aligned}
$$

if $\left(c_{1}, c_{2}\right) \in C^{2}\left(G_{0}\right)$. Here, the number $I\left(c_{1}, c_{2}\right)$ is defined by the equation of (6.4). The inequality in (6.4) follows from (6.2) since the straight lines

$$
\tilde{y}_{r}(s)=\left(D\left(c_{r}\right)-s N\left(c_{r}\right)\right) /\left|c_{r}\right|, \quad s \in \mathbb{R}, r=1,2
$$

intersect at $\left(\tilde{s}, I\left(c_{1}, c_{2}\right)\right) \in \mathbb{R}^{2}$ where

$$
\tilde{s}=\frac{\left|c_{2}\right| D\left(c_{1}\right)-\left|c_{1}\right| D\left(c_{2}\right)}{\left|c_{2}\right| N\left(c_{1}\right)-\left|c_{1}\right| N\left(c_{2}\right)} .
$$

On the other hand, using our Theorem 5 , there exist directed cycles $\beta$ and $\beta^{\prime}$ in $Z$ which satisfy (4.15)-(4.16) for a maximal point $s^{*}$ of $\varphi$. Hence we can choose the cycles $z_{r}=z_{r}^{+} \cup z_{r}^{-} \in Z_{0}, r=1,2$, such that

$$
\begin{array}{llll}
\bar{z}_{1}=\beta & \text { and } & z_{1}^{+}=\beta \cap E_{1}^{s^{*}} & \text { which implies } N\left(z_{1}\right)=n_{1 s^{*}}(\beta) \\
\bar{z}_{2}=\beta^{\prime} & \text { and } & z_{2}^{+}=\beta^{\prime} \cap E_{1}^{s^{*}} \backslash E_{3}^{s^{*}} & \text { which implies } N\left(z_{2}\right)=n_{2 s^{*}}\left(\beta^{\prime}\right) .
\end{array}
$$

Applying (4.15) and (6.1) leads to

and thus to

$$
\left|z_{r}\right| \varphi\left(s^{*}\right)=D\left(z_{r}\right)-s^{*} N\left(z_{r}\right), \quad r=1,2
$$

$$
\varphi\left(s^{*}\right)=I\left(z_{1}, z_{2}\right) \quad \text { unless } N\left(z_{1}\right)=N\left(z_{2}\right)=0 \text {. }
$$


Combining (6.3)-(6.6) completes the proof of the following explicit characterization of the infimum $\alpha$ of the Symmetric Scaling Problem 3 which is due to Rothblum and Schneider [18; Theorem 6].

Theorem 6. Under the above setting and notations,

$$
\log \alpha=-2 \min \left\{\min _{c \in C^{0}\left(G_{0}\right)} \frac{D(c)}{|c|} ; \min _{\left(c_{1}, c_{2}\right) \in C^{2}\left(G_{0}\right)} I\left(c_{1}, c_{2}\right)\right\} .
$$

Example. Let $E_{0}:=\{(1,1),(1,2),(1,3),(2,3),(3,2)\}$,

$$
\log A:=\left(a_{i j}\right):=\left(\begin{array}{ccc}
1 & 2 & 4 \\
* & * & 1 \\
* & 2 & *
\end{array}\right) .
$$

We apply Algorithm 3 starting with $s:=0$. Then we obtain the following values $s=s_{L U}$ and matrices $d^{s}$ :

$$
\begin{aligned}
& s=0: d^{s}=\left(\begin{array}{rrr}
-1 & 2 & 4 \\
-2 & * & -2 \\
-4 & -1 & *
\end{array}\right) . \\
& \varphi(s)=-3 / 2 ; \beta_{s}=\{(2,3),(3,2)\},
\end{aligned}
$$

$n_{1 s}=n_{2 s}=-2, L:=0, s_{L V}=3 / 2$

$$
\begin{gathered}
s=3 / 2: d^{s}=\left(\begin{array}{ccc}
-1 / 2 & 1 / 2 & 5 / 2 \\
-1 / 2 & * & -1 / 2 \\
-5 / 2 & 1 / 2 & *
\end{array}\right) \\
\varphi(s)=-5 / 6 ; \beta_{s}=\{(1,2),(2,3),(3,1)\},
\end{gathered}
$$

$n_{1 s}=1, n_{2 s}=-1$. Hence, $s^{*}=3 / 2$ is a maximal point of $\varphi$, and

$$
\varphi\left(s^{*}\right)=-5 / 6, \quad m^{*}=2 / 3, \quad M^{*}=7 / 3, \quad \log \alpha=5 / 3 .
$$

Step 2 of Algorithm 3. $s^{*}=3 / 2$

$$
\begin{gathered}
d^{*}=\left(d_{i j}^{s^{*}}-\varphi\left(s^{*}\right)\right)=\left(\begin{array}{rcc}
1 / 3 & 4 / 3 & 10 / 3 \\
1 / 3 & * & 1 / 3 \\
-5 / 3 & 4 / 3 & *
\end{array}\right) \\
x_{1}^{*}=-5 / 3, \quad x_{2}^{*}=-1 / 3, \quad x_{3}^{*}=0, \\
\log \left(X^{*} A X^{*-1}\right)=\left(x_{i}^{*}+a_{i j}-x_{j}^{*}\right)=\left(\begin{array}{ccc}
1 & 2 / 3 & 7 / 3 \\
* & * & 2 / 3 \\
* & 7 / 3 & *
\end{array}\right) .
\end{gathered}
$$

The directed cycles of Theorem 5 are

$$
\beta=\beta^{\prime}=\{(1,2),(2,3),(3,1)\},
$$


and the minimum in Theorem 6 is attained by $\left(z_{1}, z_{2}\right) \in C^{2}\left(G_{0}\right)$ where

$$
\begin{array}{lll}
z_{1}^{+}=\{(1,2),(2,3)\}, & z_{1}^{-}=\{(1,3)\}, & D\left(z_{1}\right)=-1, \\
z_{2}^{+}=\{(1,2)\}, & z_{2}^{-}=\{(3,2),(1,3)\}, & D\left(z_{2}\right)=-4
\end{array}
$$

and $N\left(z_{1}\right)=1, N\left(z_{2}\right)=-1$.

\section{Asymmetric Scaling}

Let $A=\left(A_{i j}\right) \neq 0$ be a non-negative $m \times n$-matrix. The matrices of the form $X A Y$ with $X \in D_{m}^{+}$and $Y \in D_{n}^{+}$are called asymmetric scalings of $A$. The measure of a asymmetric scaling is given by

where $E_{0}:=\left\{(i, j): A_{i j}>0\right\}$.

$$
\gamma(X, Y)=\frac{\max _{(i, j) \in E_{0}}(X A Y)_{i j}}{\min _{(k, l) \in E_{0}}\left(X \overline{A Y)_{k l}}\right.}
$$

Problem 4. Find the infimum

$$
\gamma:=\inf \left\{\gamma(X, Y): X \in D_{m}^{+}, Y \in D_{n}^{+}\right\}
$$

and diagonal matrices $X^{*} \in D_{m}^{+}, Y^{*} \in D_{n}^{+}$with $\gamma\left(X^{*}, Y^{*}\right)=\gamma$.

We put $a_{i j} ;=\log A_{i j},(i, j) \in E_{0}$, and

$$
\begin{aligned}
& x_{i}:=-\log X_{i}, \quad i=1, \ldots, m, \quad \text { if } X=\operatorname{diag}\left(X_{1}, \ldots, X_{m}\right) \in D_{m}^{+}, \\
& y_{j}:=-\log Y_{j}, \quad j=1, \ldots, n, \quad \text { if } Y=\operatorname{diag}\left(Y_{1}, \ldots, Y_{n}\right) \in D_{n}^{+} .
\end{aligned}
$$

Taking the logarithm of (7.1) leads to

$$
\log \gamma(X, Y)=\max _{(i, j) \in E_{0}}\left(a_{i j}-x_{i}-y_{j}\right)-\min _{(i, j) \in E_{0}}\left(a_{i j}-x_{i}-y_{j}\right) .
$$

Hence, the Scaling Problem 4 is equivalent to

Problem 4'. Find the infimum

$$
\frac{1}{2} \log \gamma=\inf _{x_{i}, y_{j}} \max _{(i, j) \in E_{0}}\left|a_{i j}-x_{i}-y_{j}\right|
$$

and numbers $x_{i}^{*}, i=1, \ldots, m$, and $y_{j}^{*}, j=1, \ldots, n$, for which the infimum is achieved.

Meanwhile, many people have studied the Problems 4 and $4^{\prime}$ or related topics. We do not intend to survey these results, but have listed a few of these articles in the References below. We only want to point out that the Problems 4 and $4^{\prime}$ are equivalent to the minimum cycle mean problem in a properly chosen weighted directed graph $\tilde{G}$. More detailed, we shall prove Theorem 7 below by introducing $\tilde{G}$ as follows. We put

$$
\begin{aligned}
\tilde{V} & :=\{1,2, \ldots, m+n\}, \\
\tilde{E} & :=\left\{(i, m+j) ;(m+j, i):(i, j) \in E_{0}\right\} \subseteq V \times V,
\end{aligned}
$$


and define the weight function $\tilde{d}: \tilde{E} \rightarrow \mathbb{R}$ by

$$
\tilde{d}_{i, m+j}:=a_{i j}, \quad \tilde{d}_{m+j, i}:=-a_{i j}, \quad(i, j) \in E_{0} .
$$

Theorem 7. Let $\lambda^{*}$ denote the minimum cycle mean of the weighted directed graph $\tilde{G}=(\tilde{V}, \tilde{E}, \tilde{d})$. Then,

$$
\frac{1}{2} \log \gamma=-\lambda^{*}
$$

and the numbers $\left(x_{1}, \ldots, x_{m+n}\right) \in \mathbb{R}^{m+n}$ are a solution of

if and only if

$$
x_{k}+\tilde{d}_{k l}-x_{l} \geqq \lambda^{*}, \quad(k, l) \in \tilde{E}
$$

Proof. The inequalities (7.3) are equivalent to

and hence to

$$
\max _{(i, j) \in E_{0}}\left|a_{i j}+x_{i}-x_{j}\right|=\frac{1}{2} \log \gamma
$$

That already concludes the proof of Theorem 7 since, by" Corollary $1, \lambda^{*}$ is the largest number for which (7.3) has a solution $x \in \mathbb{R}^{m+n}$.

Theorem 7 shows that any algorithm which solves the minimum cycle mean problem is also applicable to the Problems 4 and $4^{\prime}$. For instance, if we apply our Algorithm 1 of Sect. 3, we obtain the recurrences

and

$$
x_{i}(k):=\max \left\{x_{i}(k-1) ; a_{i j}-y_{j}(k-1): j=1, \ldots, n\right\}, \quad i=1, \ldots, m,
$$

$$
y_{j}(k):=\min \left\{y_{j}(k-1) ; a_{i j}-x_{i}(k-1): i=1, \ldots, m\right\}, \quad j=1, \ldots, n,
$$

which we have to compute for $k=1,2, \ldots, m+n$ until we can apply (3.9) for the computation of $\lambda^{*}$.

In [10; Algorithm 1] however, the recurrence (7.7a) is the same, but (7.7b) is replaced by

$$
y_{j}(k):=\min \left\{y_{j}(k-1) ; a_{i j}-x_{i}(k): i=1, \ldots, m\right\}, \quad j=1, \ldots, n,
$$

using the latest value of the $x_{i}$.

But then, we only need to compute $(7.7 \mathrm{a}),(7.7 \mathrm{~b})^{\prime}$ for $k=1, \ldots, n$, which is an essential improvement.

\section{References}

1. Aumann, G.: Über approximative Nomographie II. Bayer. Akad. Wiss. Math.-Natur, Kl. S.B., 103-109 (1959)

2. Bauer, F.L.: Optimally scaled matrices. Numer. Math. 5, 73-87 (1963)

3. Bauer, F.L.: Remarks on optimally scaled matrices. Numer. Math. 13, 1-3 (1969) 
4. Cheney, E.W., Golitschek, M.v.: On the algorithm of Diliberto and Straus for approximating bivariate functions by univariate ones. Numer. Functional Analysis and Optimization 1, 341363 (1979)

5. Cheney, E.W., Golitschek, M. v.: The best approximation of bivariate functions by separable functions. In: Topological Methods in Nonlinear Functional Analysis, pp. 125-136 (S.P. Singh, S. Thomeier, B. Watson, eds.), Contemporary Mathematics, Vol. 21. Americ. Math. Soc., 1983

6. Cheney, E.W.: Best approximation of multivariate functions. 4th Texas Symp. on Approximation Theory, College Station, January 10-14, 1983. Academic Press (L.L. Schumaker, ed.), in press

7. Collatz, L.: Approximation by functions of fewer variables. In: Conference on the theory of ordinary and partial differential equations. Held in Dundee, Scotland, March 28-31, 1972 (W.N. Everitt, B.D. Sleemann, eds.), Lecture Notes in Mathematics 280, pp. 16-31, Berlin, Heidelberg, New York: Springer 1972

8. Diliberto, S.P., Straus, E.G.: On the approximation of a function of several variables by the sum of functions of fewer variables. Pacific J. Math. 1, 195-210 (1951)

9. Fulkerson, D.R., Wolfe, P.: An algorithm for scaling matrices. SlAM Rev. 4, 142-146 (1962)

10. Golitschek, M.v.: An algorithm for scaling matrices and computing the minimum cycle mean in a digraph. Numer. Math. 35, 45-55 (1980)

11. Golitschek, M.v.: Optimal cycles in doubly weighted graphs and approximation of bivariate functions by univariate ones. Numer. Math. 39, 65-84 (1982)

12. Golitschek, M.v., Rothblum, U.G., Schneider, H.: A conforming decomposition theorem, a nonlinear theorem of the alternative, and scalings of matrices satisfying lower and upper bounds. Math. Programming (in press)

13. Golorab, M.: Approximation by functions of fewer variables. In': Symposium on Numerical Approximation (R. Langer, ed.), pp. 275-327, Madison: University of Wisconsin Press 1959

14. Karp, R.M.: A characterization of the minimum cycle mean in a digraph. Discrete Math. 23, 309-311 (1978)

15. Lawler, E.L.: Combinatorial Optimization: Networks and Matroids, New York: Holt, Rinehart and Winston 1976

16. Light, W.A., Cheney, E.W.: On the approximation of a bivariate function by the sum of univariate functions. J. Approximation Theory 29, 305-322 (1980)

17. Rivlin, T.J., Sibner, R.J.: The degree of approximation of certain functions of two variables by the sum of functions of one variable. Amer. Math. Monthly 72, 1101-1103 (1965)

18. Rothblum, U.G., Schneider, H.: Characterizations of optimal scalings of matrices. Math. Programming 19, 121-136 (1980)

19. Saunders, B.D., Schneider, H.: Flows on graphs applied to diagonal similarity and diagonal equivalence of matrices. Discrete Math. 24, 202-220 (1978)

20. Saunders, B.D., Schneider, H.: Cones, graphs and optimal scalings of matrices. Linear and Multilinear Algebra 8, 121-135 (1979)

21. Sprecher, D.A.: On best approximations of functions of two variables. Duke Math. J. 35, 391$397(1968)$

22. Tomlin, J.A.: On scaling linear programming problems. Mathematical Programming 4, $146-$ 166 (1975)

23. Wille, H., Gewald, K., Weber, H.D.: Netzplantechnik. Bd. 1, München-Wien: Oldenbourg 1966

Received June 7,1983/September 7, 1983 